Original article

\title{
Elevated circulating endothelial cell-derived microparticle levels in patients with liver cirrhosis: a preliminary report
}

\author{
Jolanta Zuwała-Jagiełło', Krzysztof Adam Simon ${ }^{2,3}$, Monika Pazgan-Simon ${ }^{4}$ \\ 'Department of Pharmaceutical Biochemistry, Wroclaw Medical University, Wroclaw, Poland \\ ${ }^{2}$ Division of Infectious Disease and Hepatology, Wroclaw Medical University, Wroclaw, Poland \\ ${ }^{3}$ I Department of Infectious Diseases, Regional Specialist Hospital, Wroclaw, Poland \\ 4J. Gromkowski Regional Specialist Hospital, Wroclaw, Poland
}

\begin{abstract}
Aim of the study: To determine plausible associations between liver cirrhosis and circulating endothelial cell-derived microparticles (EMPs), vascular endothelial growth factor (VEGF) levels and plasma nitric oxide (NO) metabolites.

Material and methods: Sixty patients with cirrhosis and 20 healthy control subjects were enrolled in the study. Circulating EMPs from platelet-poor plasma samples were examined by flow cytometry. These microparticles were categorized into endothelial cell-derived activated MPs (EMP-ac) (CD31+ CD42b- AN-V) and endothelial cell-derived apoptotic MPs (EMP-ap) (CD31+ $\left.\mathrm{CD} 42 \mathrm{~b}^{-} \mathrm{AN}-\mathrm{V}^{+}\right)$. Plasma VEGF levels were measured by enzyme-linked immunosorbent assay. Plasma NO metabolites $\left(\mathrm{NO}_{x}^{-}\right)$levels were determined using a Greiss reaction method.

Results: Compared with the healthy control subjects, the patients with cirrhosis showed a significant increase in plasma levels of both phenotypes of EMPs. When the presence of ascites was considered, the plasma levels of EMP-ap were higher $(p<0.01)$, as well as $\mathrm{NO}_{\mathrm{\gamma}}^{-}(p<0.05)$. EMP-ap positively correlated with VEGF level in all cirrhotic patients and this correlation was stronger in decompensated cirrhotic patients. In multivariate logistic regression analysis, the independent factors associated with the presence of ascites were high EMP-ap levels and elevated VEGF levels.

Conclusions: Elevated plasma levels of EMP-ap in addition to high levels of VEGF might be considered as valuable parameters for predicting the occurrence of ascites in cirrhotic patients.
\end{abstract}

Key words: microparticle, vascular permeability, liver cirrhosis.

\section{Address for correspondence}

Jolanta Zuwała-Jagiełło, J. Gromkowski Regional Specialist Hospital, 5 Koszarowa St., 51-149 Wroclaw, Poland, e-mail: jagiellodr@interia.pl

\section{Introduction}

Endothelial cells play an active role in the modifications of the circulatory status of cirrhotic patients [1]. The circulatory changes are more evident in decompensated cirrhosis, such as those represented by the presence of ascites [2,3]. However, the extent and molecular mechanisms governing plasma extravasation and formation of ascites in cirrhosis are unknown.

Endothelial cells are the primary barrier to vascular extravasation, thus regulating the passage of molecules by a variety of mechanisms that are chiefly regulated by vascular endothelial growth factor (VEGF) type A [4]. By interacting with vascular endothelial growth factor receptor 2, VEGF not only induces angiogenesis but also disrupts vascular barrier function in diseased tissues $[5,6]$. The list of substances that increase vascular permeability in vivo has recently been enlarged by endothelial cell-derived microparticles (EMPs). The term EMPs usually refers to vesicles $0.1-1 \mu \mathrm{m}$ in diameter produced by budding from the plasma membrane. Another feature of microparticles (MPs) is the exposure of phosphatidylserine (PS) at their surface, which can be detected by labeling of annexin- $\mathrm{V}$ using 
flow cytometry analysis. The phenotypic assessment of membrane surface constituents can provide information on the functional status of the endothelium from which they were released. In vitro studies have shown that endothelial cells release different EMPs in response to activation and apoptosis [7]. Furthermore, the exposure of specific receptors at their surface indicates that circulating EMPs behave as bio-messengers linking angiogenesis and inflammation [8]. Indeed, accumulating published data demonstrate that numbers of circulating EMPs are increased in a wide range of diseases, particularly cardiovascular diseases [9-11], but also in chronic hepatitis [12]. The results of a previous study strongly suggest that circulating EMPs represent not only a measure of endothelial cell injury but also a source of vascular permeability factors [13].

Enhanced vascular permeability in the hepatic and mesenteric vascular beds contributes to the formation of ascites in cirrhosis [14], and EMPs might impair endothelium-mediated vasodilation and directly increase endothelial permeability [15]. Endothelial microparticles have only been detected in the ascites of patients with ovarian and colorectal cancer $[16,17]$. In this context, the association between EMPs and the vascular permeability in cirrhotic patients could represent one of the mechanisms governing formation of ascites in this population.

Nitric oxide (NO) is a potent inducer of increased membrane permeability in the vascular endothelium possibly contributing to the accumulation of ascites [18, 19]. Plasma nitrate and nitrite $\left(\mathrm{NO}_{\mathrm{x}}^{-}\right.$, $\mathrm{NO}$ metabolites) have been found variably increased in cirrhosis, having associations with ascites, Child-Pugh score and portal hypertension [20]. On the other hand, EMPs were thought to induce NO synthesis through endothelial nitric oxide synthase [15]. Therefore, it is plausible that circulating EMP-mediated increases in the nitric oxide metabolites in plasma are related to the magnitude of vascular permeability and hence to ascites in cirrhosis.

To clarify the role of circulating EMPs, plasma NO metabolite levels and VEGF level in the development of decompensated cirrhosis, we investigated whether circulating EMPs are altered in cirrhotic patients, and their relationship with plasma NO metabolite levels and VEGF level.

\section{Material and methods}

\section{Patients and study design}

This study was performed in 60 patients with liver cirrhosis admitted to the First Department of Infectious Diseases and Division of Infectious Disease and Hepatology for evaluation. The patients with cirrho- sis were divided into two groups in function of the presence ( 23 patients) or the absence ( 37 patients) of ascites. The control group (20 subjects) was composed of healthy subjects age- and sex-matched with the patients. Blood samples were collected in the Department of Physiology and Biochemistry, University of Physical Education in Wroclaw. The absence of evidence of infection was confirmed by anamnesis, physical exploration and laboratory tests (absence of increase in leucocyte count, normal values of globular sedimentation rate and C-reactive protein and normal urine elemental analysis).

Inclusion criteria were: histological or clinical diagnosis of cirrhosis, no evidence of metabolic, toxic or autoimmune liver disease and at least 1 year of alcohol abstinence. Diagnosis of cirrhosis was established according to histological criteria when liver biopsy was performed, or by the combination of clinical, biochemical, ultrasound, CT and GI endoscopy imaging data consistent with such a diagnosis. Patients were grouped according to the Child-Pugh classification. Three biochemical variables (serum albumin, bilirubin, and prothrombin time [international normalized ratio, INR]) in addition to the presence or absence of ascites determine the Child-Pugh score. Presence of ascites was assessed by ultrasonography. Bacterial infection was ruled out by clinical history, physical examination, differential and total white blood cell count, analysis and culture of urine, thorax X-ray and by the culture and white blood cell count of ascetic fluid in patients with ascites.

Exclusion criteria were co-existing diseases such as diabetes mellitus, chronic kidney disease, cardiovascular disease, cardiac decompensation, hepatocellular carcinoma, or heart failure, gastrointestinal bleeding, bacterial infection, and blood transfusion within the previous two weeks. Patients received no diuretic, antibiotic, or vasoactive drug (nitrates, $\beta$-blockers), during the eight days before inclusion in the study.

The consent of the Bioethics Committee of the Wroclaw Medical University was obtained and all patients were informed about the character of analyses made. Studies were conducted in compliance with the ethical standards formulated in the Helsinki Declaration of 1975 (revised in 1983).

\section{Assessment of plasma circulating endothelial microparticles}

The circulating microparticles (MPs) were categorized into endothelial-derived activated MPs (EMP-ac) $\left(\mathrm{CD} 31^{+} \mathrm{CD}_{2} \mathrm{~b}^{-} \mathrm{AN}-\mathrm{V}^{-}\right)$and endothelial-derived apoptotic MPs (EMP-ap) $\left(\mathrm{CD} 31^{+} \mathrm{CD} 42 \mathrm{~b}^{-} \mathrm{AN}-\mathrm{V}^{+}\right)$based on a previous report [21] with some modifications. 
Peripheral blood was collected in acid citrate dextrose vacutainer tubes, using a 21-gauge needle. The first $3 \mathrm{ml}$ of blood were discarded to avoid contamination with EMPs due to vascular injury [22]. The peripheral blood $(1.5 \mathrm{ml})$ was centrifuged at $2500 \mathrm{~g}$ at $4^{\circ} \mathrm{C}$ for $15 \mathrm{~min}$ without acceleration or break to prepare platelet-poor plasma. The $250 \mu \mathrm{l}$ plasma samples were thawed and centrifuged for $10 \mathrm{~min}$ at $19700 \mathrm{~g}$ at $4^{\circ} \mathrm{C}$ and then collected for investigation of microparticles smaller than $1.0 \mu \mathrm{m}$. Size calibration was conducted with $1.0 \mu \mathrm{m}$ beads. The MP pellet was resuspended with $150 \mu \mathrm{l}$ of Annexin- $\mathrm{V}$ binding buffer (BD Biosciences). All buffers were sterile-filtered with a $0.2 \mu \mathrm{m}$ filter. Then $100 \mu \mathrm{l}$ of MPs were incubated in a Trucount tube (BD Biosciences) with fluorescent monoclonal antibodies: anti-CD31- PE, Anti-annexin-V-FITC, and anti-CD42b-PE (BD Biosciences, U.S.). The samples were incubated in the dark for $15 \mathrm{~min}$ at room temperature. The samples were then analyzed on a FC500 flow cytometer (Beckman Coulter) after $400 \mu \mathrm{l}$ of Annexin- $\mathrm{V}$ binding buffer was added. The absolute count of MPs was measured setting the stop condition for Trucount beads at 10000 events. Fluorescence minus one controls and non-stained samples were used to discriminate true events from noise, and to increase the specificity for EMP detection. The contamination of $\mathrm{CD} 31^{+} \mathrm{CD} 42 \mathrm{~b}^{-}$EMPs with $\mathrm{CD} 45^{+} \mathrm{CD} 31^{+} \mathrm{CD} 42 \mathrm{~b}^{-}$ leukocyte MPs was tested using the pan-leukocyte marker CD45. The amount of $\mathrm{CD} 45^{+} \mathrm{CD} 31^{+} \mathrm{CD} 42 \mathrm{~b}^{-}$leukocyte MPs was low (4.6\% in platelet-poor plasma). Values are reported as counts per microliter.

\section{Measurement of plasma vascular endothelial growth factor}

Because platelets are a potential source of VEGF, we measured VEGF level in platelet-poor plasma. Platelet-poor plasma levels of VEGF were assayed with enzyme-linked immunosorbent assay (ELISA) kits (R\&D Systems Inc., Minneapolis, MN, USA) according to the manufacturer's instructions.

\section{Measurement of plasma nitric oxide metabolites $\left(\mathrm{NO}_{\times}^{-}\right)$}

NO levels in plasma are short lived and were thus evaluated by its stable metabolites nitrate $\left(\mathrm{NO}_{3}^{-}\right)$and nitrite $\left(\mathrm{NO}_{2}^{-}\right)$using the Griess reaction. A commercial kit was used with modifications adapted for plasma samples [23] (Sigma 23479, nitrate/nitrite Assay Kit Colorimetric, Sigma-Aldrich, St. Louis, MO, USA). Briefly, samples were incubated with nitrate reductase to reduce nitrate to nitrite. Griess reagent was then added and absorbance was measured at $570 \mathrm{~nm}$ to evaluate total nitrite levels (Bio-Rad 680 microplate reader, Bio-Rad Laboratories, CA, USA). Background absorbance was measured for each sample and subtracted from total values.

\section{Statistical analysis}

Continuous variables are expressed as median (interquartile range $[\mathrm{IQR}]$ ) and categorical variables as number (percentage). Unpaired data were analyzed by the independent samples $t$-test or the Mann-Whitney test. Paired data were analyzed by the paired Student $t$-test or Wilcoxon test. All reported $p$-values are twotailed, with values $<0.05$ considered significant. Correlation analysis between stochastically independent variables were performed by the Pearson correlation test. Multiple logistic regression analysis was used to identify factors related to the presence of ascites. Variables that achieved statistical significance in the univariate analysis ( $p$-value $<0.05$ ) were subsequently included in a logistic regression analysis. Selection of variables was based on a stepwise regression analysis using a forward selection method.

\section{Results}

\section{Characteristics of the participating patients}

Table 1 shows the clinical and routine analytical features of the patients included in the study. A total of 60 cirrhotic patients were included in the study. In more than half the patients, liver cirrhosis was secondary to chronic hepatitis $\mathrm{C}$ virus infection, whereas alcohol consumption was the second cause of cirrhosis (eleven cases, 18\%); in the rest of the patients (seven cases, $12 \%$ ), both alcohol and hepatitis $C$ virus were considered the cause of cirrhosis. The patients were divided into two groups: 37 cirrhotic patients without ascites and 23 cirrhotic patients with ascites. Eleven (48\%) patients had refractory ascites. No differences were found in age, gender ratio, or cause of liver disease between the ascites and the non-ascites group (Table 2). As expected, liver and/or renal function indices (Child-Pugh score or creatinine concentration) were significantly higher in patients with ascites $(p<0.01)$ (Table 2$)$.

\section{Circulating EMP-ap (CD31 + CD42 $\left.\mathrm{b}^{-} \mathrm{AN}-\mathrm{V}^{+}\right)$and EMP-ac (CD31 $\left.{ }^{+} \mathrm{CD}_{2} \mathrm{~b}^{-} \mathrm{AN}-\mathrm{V}^{-}\right)$plasma levels in patients with liver cirrhosis and healthy controls}

We analyzed 60 patients ( 34 males/26 females; median age: 57, IQR: $42-71$ years) with liver cirrhosis. The plasma levels of circulating EMP-ap (CD31 $\left.\mathrm{CD}_{2} \mathrm{~b}^{-} \mathrm{AN}^{-} \mathrm{V}^{+}\right)$and EMP-ac $\left(\mathrm{CD} 31^{+} \mathrm{CD}^{-} \mathrm{b}^{-} \mathrm{AN}^{-\mathrm{V}^{-}}\right)$ 
Table 1. Clinical and biochemical parameters in cirrhotic patients and healthy controls

\begin{tabular}{|c|c|c|}
\hline & $\begin{array}{l}\text { Healthy } \\
\text { controls }\end{array}$ & $\begin{array}{l}\text { Cirrhotic } \\
\text { patients }\end{array}$ \\
\hline$n$ & 20 & 60 \\
\hline Male : female ratio & $11 / 9$ & $34 / 26$ \\
\hline Age (years) & $55(45-66)$ & $57(42-71)$ \\
\hline \multicolumn{3}{|l|}{ Etiology of cirrhosis, $n(\%)$} \\
\hline Alcohol & - & $11(18)$ \\
\hline Hepatitis C virus & - & $42(70)$ \\
\hline Other & - & $7(12)$ \\
\hline Ascites (+/-) & - & $23 / 37$ \\
\hline Child-Pugh score & - & $8(4-10)$ \\
\hline Albumin $(g / d l)$ & $4.8(3.6-5.7)$ & $2.7(1.9-3.7)^{* *}$ \\
\hline Bilirubin (mg/dl) & $0.7(0.6-0.9)$ & $3.5(1.5-6.0)^{*}$ \\
\hline INR & $0.8-1.2$ & $1.35(0.81-2.06)$ \\
\hline Creatinine (mg/dl) & $0.8(0.7-1.0)$ & $0.95(0.6-1.7)^{*}$ \\
\hline Serum urea nitrogen (mg/dl) & $13.8(5.6-18.8)$ & $18.8(3.7-23.4)^{*}$ \\
\hline Serum sodium (mmol/l) & 140 (138-141) & $137(129-142)$ \\
\hline Mean arterial blood pressure $(\mathrm{mg} \mathrm{Hg})$ & - & $80(76.6-85)$ \\
\hline $\begin{array}{l}\text { EMP-ap } \\
\text { CD31+CD42b-AN-V+ } \\
\text { (counts/ } / \mu l)\end{array}$ & $0.52(0.24-1.05)$ & $5.4(0.31-9.8)^{\star \star}$ \\
\hline  & $5.6(3.8-10.8)$ & $10.5(2.15-23.6)$ \\
\hline
\end{tabular}

Continuous variables are expressed as median (interquartile range) and categorical variables as number (percentage). Significance levels between groups: ${ }^{*} p<0.05$,

${ }^{* *} p<0.01$ vs. healthy controls.

EMP-ap - endothelial-derived apoptotic microparticles $\left(C D 31^{+} C D 42 b^{-} A N-V^{+}\right)$, EMP-ac endothelial-derived activated microparticles (CD31+ $\left.C D 42 b^{-} A N-V\right)$, INR - international normalized ratio

in the group of patients with cirrhosis and subjects belonging to the control group are presented in Table 1. Circulating EMP-ap and EMP-ac were also checked for correlations with selected biochemical markers of liver function (albumin, bilirubin concentration, INR) and injury (aminotransferases).

Plasma levels of circulating EMP-ap were significantly higher in all cirrhotic patients than in healthy controls $(n=60$, median: 5.4 counts/ $\mu$ l, IQR: 0.31-9.8 counts $/ \mu \mathrm{l} ; p<0.01)$ (Table 1$)$. In the control group, the plasma circulating EMP-ap levels were similar to those in healthy controls in other studies [24]. The concentration of circulating EMP-ac was measured in all 60 patients with cirrhosis with a median of 10.5 counts/ $\mu \mathrm{l}$ (IQR: 2.15-23.6 counts/ $\mu$ l).
According to an analysis relating circulating EMPac and EMP-ap level to the presence of complications of cirrhosis for patients as indicated by the hypoalbuminemia, hyperbilirubinemia, and prolonged INR, there were no significant differences. In the study group, no significant correlations were also observed between EMP-ac and EMP-ap and biochemical markers of liver injury (not reported in detail).

\section{Clinical and biochemical characteristics of patients with liver cirrhosis according to the presence of ascites}

The biochemical and clinical characteristics of healthy controls and cirrhotic patients both with and without ascites are shown in Table 2. Distribution of sex was similar among groups. By design, bilirubin, creatinine levels, and Child-Pugh score were higher in cirrhotic patients with ascites than in patients without ascites, and serum urea nitrogen levels were higher in cirrhotic patients with ascites than in the healthy controls.

Circulating EMP-ap levels were significantly different between the non-ascites and ascites group $(n=23$, me-

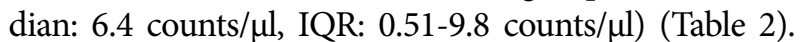
Moreover, EMP-ap production in refractory ascites $(n=11)$ was significantly higher compared with the non-ascites group (median: 7.9 counts/ $\mu$ [IQR: 5.6-9.8 counts/ $\mu \mathrm{l}$ ] vs. 3.2 counts/ $\mu \mathrm{l}$ [IQR: $0.31-5.3$ counts $/ \mu$ ] , respectively; $p<0.001$ ).

The plasma circulating EMP-ac level in cirrhotic patients with ascites was significantly higher than that in healthy controls $(p<0.05)$ (Table 2). The patients without ascites showed high circulating levels of EMP-ac but the median of this group was not significantly higher than the healthy controls $(8.5$ counts/ $\mu \mathrm{l}$ vs. 5.6 counts $/ \mu \mathrm{l}$, respectively) (Table 2). The difference between non-ascites and ascites groups with respect to EMP-ac was not statistically significant $(p>0.05)$ (Table 2$)$.

Plasma levels of both phenotypes of EMPs were increased in patients with a more severe clinical state of liver cirrhosis; however, median differences in levels of EMPs among the Child-Pugh classes were not statistically significant (data not shown).

The median nitrate + nitrite $\left(\mathrm{NO}_{\mathrm{x}}^{-}\right)$levels were higher in the cirrhotic patients with ascites than in the healthy controls $(117.0 \mu \mathrm{mol} / \mathrm{l}$ vs. $51.0 \mu \mathrm{mol} / \mathrm{l}$, respectively; $p<0.01$ ) (Table 2). A significant difference was observed in plasma levels of $\mathrm{NO}_{x}^{-}$between cirrhotic patients with and without ascites $(p<0.01)$. However, $\mathrm{NO}_{\mathrm{x}}^{-}$levels in the non-ascites group were similar to those in the healthy controls.

Vascular endothelial growth factor plasma levels were increased in the non-ascites and ascites group, with high- 
Table 2. Clinical and biochemical characteristics of patients with liver cirrhosis according to the presence of ascites

\begin{tabular}{|c|c|c|c|}
\hline & Healthy controls & Cirrhotic patients without ascites & Cirrhotic patients with ascites \\
\hline$n$ & 20 & 37 & 23 \\
\hline Male : female ratio & $11 / 9$ & $19 / 18$ & $13 / 10$ \\
\hline Age (years) & $55(45-66)$ & $57(47-68)$ & $56(42-71)$ \\
\hline \multicolumn{4}{|l|}{ Etiology of cirrhosis, $n(\%)$} \\
\hline Alcohol & - & $7(19)$ & $4(17)$ \\
\hline Hepatitis C virus & - & $24(65)$ & $18(78)$ \\
\hline Other & - & $2(16)$ & $5(5)$ \\
\hline Refractory ascites, $n(\%)$ & & - & $11(48)$ \\
\hline Child-Pugh class (A, B, C) & - & $26 / 11 / 0$ & $0 / 13 / 10$ \\
\hline Child-Pugh score & - & $6(4-7)$ & $9(8-10)^{++}$ \\
\hline Albumin (g/dl) & $4.8(3.6-5.7)$ & $2.8(1.9-3.7)^{* *}$ & $2.6(2.1-3.1)^{* *}$ \\
\hline Bilirubin (mg/dl) & $0.7(0.6-0.9)$ & $2.9(1.5-5.0)^{*}$ & $4.3(2.1-6.0)^{*+}$ \\
\hline Creatinine (mg/dl) & $0.80(0.7-1.0)$ & $0.79(0.6-1.4)$ & $1.2(0.8-1.7)^{* *++}$ \\
\hline Serum urea nitrogen (mg/dl) & $14.0(5.6-18.8)$ & $15.6(3.7-19.1)$ & $23.5(8.9-23.4)^{*}$ \\
\hline $\begin{array}{l}\text { EMP-ap } \\
\text { CD31+CD42b-AN-V+(counts/ } \mu l)\end{array}$ & $0.52(0.24-1.05)$ & $3.2(0.31-5.3)^{\star \star}$ & $6.4(0.51-9.8)^{* \star *++}$ \\
\hline $\begin{array}{l}\text { EMP-Ac } \\
\text { CD31+CD42b-AN-V (counts/ } / \mu l)\end{array}$ & $5.6(3.8-10.8)$ & $8.5(2.15-16.2)$ & $13.8(4.2-23.6)^{*}$ \\
\hline $\mathrm{NO}_{x}^{-}\left(\mathrm{NO}_{3}+\mathrm{NO}_{2}\right)(\mu \mathrm{mol} / \mathrm{l})$ & $51.0(6.0-78)$ & $55.7(51.0-104.0)$ & $117.0(114.2-120.0)^{* *++}$ \\
\hline $\operatorname{VEGF}(p g / m l)$ & $19.3(0.0-25.1)$ & $116.8(15.0-262.5)^{* *}$ & $150.7(20.5-315.0)^{* * *}$ \\
\hline
\end{tabular}

Continuous variables are expressed as median (interquartile range; IQR) and categorical variables as number (percentage).

Significance levels between groups: ${ }^{*} p<0.05,{ }^{* *} p<0.01,{ }^{* * *} p<0.001$ v healthy controls; ${ }^{*} p<0.05,{ }^{++} p<0.01$ vs cirrhotic patients without ascites

EMP-ap - endothelial-derived apoptotic microparticles (CD31+ CD42b- AN-V+), EMP-ac - endothelial-derived activated microparticles (CD31 $\left.1^{+} C D 42 b^{-} A N-V\right)$, VEGF-endothelial growth factor, $\mathrm{NO}_{x}^{-}$- nitric oxide metabolites

er levels in the latter (Table 2). Significant correlations between VEGF levels and EMP-ap $(r=0.31, p<0.05)$ were observed among the patients with cirrhosis.

\section{Multiple regression analysis}

Based on stepwise multiple logistic regression analysis of factors (Child-Pugh classification, creatinine, EMP-ap $\left(\mathrm{CD} 31^{+} \mathrm{CD} 42 \mathrm{~b}^{-} \mathrm{AN}-\mathrm{V}^{+}\right)$, NO metabolites, and VEGF), VEGF level [odds ratio (OR): $11.593,95 \%$ confidence interval (CI): $3.742-16.024, p<0.007]$ and plasma EMP-ap level (OR: 6.285, 95\% CI: 3.837-8.887, $p<0.001)$ were found to be independent predictors of ascites formation (Table 3 ).

\section{Discussion}

In the present study, we investigated the relationship between circulating endothelial cell-derived microparticles and vascular permeability by comparing the major regulator of vascular permeability (VEGF) in cirrhotic patients showing high levels of EMPs in the presence of high plasma $\mathrm{NO}_{\mathrm{x}}^{-}$(NO metabolites) levels. The main results are summarized as follows: (a) Compared with healthy controls, all the cirrhotic patients had higher levels of EMPs, which were associated with increased VEGF. (b) When the presence of ascites was considered, the plasma levels of EMP-ap were higher, as well as the NO metabolites. (c) When the EMP-ap levels were analyzed individually in cirrhotic patients, there

Table 3. Multiple logistic regression analysis of factors associated with the presence of ascites

\begin{tabular}{lccc}
\hline & OR & 95\% Cl & $p$ value \\
\hline VEGF $(\mathrm{pg} / \mathrm{ml})$ & 11.593 & $3.742-16.024$ & 0.007 \\
\hline EMP-ap & 6.3485 & $3.858-8.887$ & 0.001 \\
$\begin{array}{l}\text { CD31 } 1^{+} \mathrm{CD} 42 \mathrm{~b}-\mathrm{AN}-V^{+} \\
\text {(counts/ } / \mathrm{ll})\end{array}$ & & & \\
\hline
\end{tabular}

VRGF - vascular endothelial growth factor, EMP-ap - endothelial-derived apoptotic microparticles (CD31+ $\left.\mathrm{CD} 42 b^{-} \mathrm{AN}-\mathrm{V}^{+}\right)$ 
was a positive association of plasma EMP-ap with the VEGF levels. (d) Multiple regression analysis showed that plasma levels of EMP-ap and the VEGF levels were significantly correlated with ascites formation.

In decompensated cirrhosis, endothelial cells are the primary barrier to vascular extravasation, thus regulating the passage of molecules by a variety of mechanisms that are chiefly regulated by the vascular endothelial growth factor [4]. Enhanced endothelial permeability is restricted to the hepatic and mesenteric vascular beds in ascites, and VEGF is the key factor in the signaling pathways regulating this dysfunction. In fact, VEGF increases vascular permeability by disrupting intercellular contacts in adherens, tight, and gap junctions [14]. However, other mechanisms may alter endothelial permeability, as measured by increased EMP production. Our results showed that EMP-ap levels in all cirrhotic patients were independently associated with the presence of ascites, and showed significant correlations with VEGF levels. Finally, the values of EMP-ap in patients with a low Child-Pugh score and absence of ascites suggest that EMP-ap might have a role in the late stages of cirrhosis by aggravating the already initiated endothelial injury.

Increased circulating EMP-ap levels, along with $\mathrm{NO}_{x}^{-}$levels in cirrhotic patients, especially higher values in the refractory ascites group, were observed in the present study. Interestingly, in vitro studies in bovine aortic endothelial cell monolayers suggest that EMPs may mediate vascular permeability effects by changes in the production of nitric oxide [15]. Since NO is a potent inducer of increased membrane permeability in the vascular endothelium, it is likely to affect both the progress and the clinical features of cirrhosis as well as the hemodynamics in cirrhotic patients [19]. Although EMPs were thought to induce NO synthesis through endothelial nitric oxide synthase [15], there was no significant correlation between plasma $\mathrm{NO}_{x}{ }^{-}$levels and the circulating EMP-ap in cirrhotic patients. Taken together, these results suggest that some other factors including EMP-ap may contribute to the accumulation of ascites.

The main limitation of this study, besides the unavoidable clinical heterogeneity and the relatively small number of patients, lies in its cross-sectional nature, unable to identify pathogenic mechanisms(s) linking EMPs, VEGF plasma levels, and development of ascites.

\section{Conclusions}

The simultaneous monitoring of plasma EMP-ap, defined by the phenotype CD $31^{+} \mathrm{CD} 42 \mathrm{~b}^{-} \mathrm{AN}-\mathrm{V}^{+}$, and VEGF can be helpful for the development of ascites control in cirrhotic patients, although the pathologic link between the two biomarkers remains unclear. Our findings emphasize the need for further investigations correlating the plasma levels of EMP-ap seen in decompensated cirrhotic patients with their clinical outcomes (for example, renal failure or septic complications) to develop prognostic parameters.

\section{Acknowledgments}

The project described was supported by a grant from Wroclaw Medical University, no. ST-759.

\section{Disclosure}

Authors report no conflict of interest.

\section{References}

1. Genesca J, González A, Mujal A, et al. Vascular endothelial growth factor levels in liver cirrhosis. Dig Dis Sci 1999; 44: 1261-1262.

2. Moore KP, Aithal GP. Guidelines on the management of ascites in cirrhosis. Gut 2006; 55 (Suppl 6): vi1-vi12.

3. Ackerman Z. Ascites in nephrotic syndrome. Incidence, patients' characteristics, and complications. J Clin Gastroenterol 1996; 22: 31-34

4. Morales-Ruiz M, Jiménez W. Neovascularization, angiogenesis, and vascular remodeling in portal hypertension. In: Portal Hypertension. Pathogenesis, Evaluation and Treatment. Sanyal AJ, Shah VH (eds.). Humana Press, Totowa 2005; 99-112.

5. Weis SM, Cheresh DA. Pathophysiological consequences of VEGF-induced vascular permeability. Nature 2005; 437: 497-504.

6. Yancopoulos GD, Davis S, Gale NW, et al. Vascular-specific growth factors and blood vessel formation. Nature 2000; 407: 242-248.

7. Jimenez JJ, Jy W, Mauro LM, et al. Endothelial cells release phenotypically and quantitatively distinct microparticles in activation and apoptosis. Thromb Res 2003; 15: 175-180.

8. Lemoinne $S$, Thabut $D$, Housset $C$, et al. The emerging roles of microvesicles in liver diseases. Nat Rev Gastroenterol Hepatol 2014; 11: 350-361.

9. VanWijk MJ, VanBavel E, Sturk A, et al. Microparticles in cardiovascular diseases. Cardiovasc Res 2003; 59: 277-287.

10. Mallat Z, Benamer H, Hugel B, et al. Elevated levels of shed membrane microparticles with procoagulant potential in the peripheral circulating blood of patients with acute coronary syndromes. Circulation 2000; 101: 841-843.

11. Morel O, Hugel B, Jesel L, et al. Circulating procoagulant microparticles and soluble GPV in myocardial infarction treated by primary percutaneous transluminal coronary angioplasty. A possible role for GPIIb-IIIa antagonists. J Thromb Haemost 2004; 2: 1118-11126.

12. Zuwala-Jagiello J, Pazgan-Simon M, Murawska-Cialowicz E, et al. FEBS EMBO 2014 Conference. Circulating endothelial microparticles in patients with chronic hepatitis $\mathrm{C}$ infection. FEBS J 2014; 281 (Suppl 1): 107.

13. Shet AS, Aras O, Gupta K, et al. Sickle blood contains tissue factor-positive microparticles derived from endothelial cells and monocytes. Blood 2003; 102: 2678-2683.

14. Melgar-Lesmes P, Tugues S, Ros J, et al. Vascular endothelial growth factor and angiopoietin-2 play a major role in the athogenesis of vascular leakage in cirrhotic rats. Gut 2009; 58: 285-292. 
15. Densmore JC, Signorino PR, Ou J, et al. Endothelium-derived microparticles induce endothelial dysfunction and acute lung injury. Shock 2006; 26: 464-471.

16. Press JZ, Reyes M, Pitteri SJ, et al. Microparticles from ovarian carcinomas are shed into ascites and promote cell migration. Int J Gynecol Cancer 2012; 22: 546-552.

17. Choi DS, Park JO, Jang SC, et al. Proteomic analysis of microvesicles derived from human colorectal cancer ascites. Proteomics 2011; 11: 2745-2751.

18. Vallance P, Moncada S. Hyperdynamic circulation in cirrhosis: a role for nitric oxide? Lancet 1991; 337: 776-778.

19. Guarner C, Soriano G, Tomas A, et al. Increased serum nitrite and nitrate levels in patients with cirrhosis: relationship to endotoxemia. Hepatology 1993; 18: 1139-1143.

20. Curgunlu A, Vural P, Canbaz M, et al. Plasma nitrate/nitrite and endothelin-1 in patients with liver cirrhosis. J Clin Lab Anal 2005; 19: 177-181.

21. van Ierssel SH, Van Craenenbroeck EM, Conraads VM, et al Flow cytometric detection of endothelial microparticles (EMP): effects of centrifugation and storage alter with the phenotype studied. Thrombosis Research 2010; 125: 332-339.

22. Goon PK, Boos CJ, Stonelake PS, et al. Detection and quantification of mature circulating endothelial cells using flow cytometry and immunomagnetic beads: a methodological comparison. Thrombosis and Haemostasis 2006; 96: 45-52.

23. Ricart-Jané D, Llobera M, López-Tejero MD. Anticoagulants and other preanalytical factors interfere in plasma nitrate/nitrite quantification by the Griess method. Nitric Oxide 2002; 6: 178-185.

24. van Ierssel SH, Van Craenenbroeck EM, Conraads VM, et al. Flow cytometric detection of endothelial microparticles (EMP): effects of centrifugation and storage alter with the phenotype studied. Thromb Res 2010; 125: 332-339. 